\title{
Tendência das internações hospitalares por complicações de aborto no Brasil, 2000-2015
}

\author{
Trends in hospitalization for abortion-related complications in Brazil, 2000-2015 \\ Tendencias en la hospitalización por complicaciones relacionadas con el aborto en Brasil, \\ 2000-2015 \\ Sofia Naira Barbosa Freitas ${ }^{1}$, Andréa Cronemberger Rufino ${ }^{1}$, Alberto Pereira Madeiro ${ }^{1 *}$.
}

\section{RESUMO}

Objetivo: Analisar a magnitude e tendências das internações hospitalares por complicações de aborto no Brasil, entre 2000 e 2015. Métodos: Estudo ecológico, de tendência temporal, com dados de 2000-2015 sobre internações hospitalares por complicações de aborto em mulheres de 15-44 anos no Brasil, regiões e estados, obtidos do DATASUS e do IBGE. Utilizou-se o método Joinpoint para avaliar a tendência das taxas de internações, com determinação da variação percentual anual (VPA) e intervalos de confiança de 95\% (IC95\%). Resultados: Entre 2000-2015, ocorreram 3.569.661 internações por complicações de aborto no Brasil. A região com maior taxa foi a Nordeste $(7,0)$ em $2000-2003$, enquanto que a Sul apresentou as menores taxas nos quatro quadriênios. Dentre os estados, Amapá $(12,2)$ e Sergipe $(11,0)$ tiveram as maiores taxas em 2000-2003, enquanto Paraíba $(3,0)$ e Rio Grande do Sul $(3,2)$ as menores. Houve tendência de redução da taxa de internações no Brasil (VPA= -4,21/IC95\% -7,23;-1,78), assim como nas regiões Nordeste (VPA=-4,21/IC95\% -7,23;-1,78) e Sudeste (VPA=-4,21/IC95\% -7,23;-1,78). As demais apresentaram tendência estável. Conclusão: Apesar da diminuição no número de internações e tendência de declínio das taxas entre 2000-2015 no país, ainda permanece alto o número de mulheres que precisam ser internadas para finalizar o aborto.

Palavras-chave: Aborto, Hospitalização, Tendências.

\section{ABSTRACT}

Objective: To analyze magnitude and trends of hospitalizations due abortion-related complications in Brazil, 2000-2015. Methods: Ecological, time series study with data from 2000-2015 on hospital admissions due to complications of abortion in women aged 15-44 years in Brazil, regions and states, obtained from DATASUS and IBGE. The Joinpoint method was used to evaluate trends of hospitalization rates, with determination of annual percent change (APC) and 95\% confidence intervals $(95 \% \mathrm{Cl})$. Results: Between 2000-2015, there were 3,569,661 hospitalizations due abortion in Brazil. The region with the highest hospitalization rate was the Northeast (7.0) in 2000-2003, while the South had lowest rates in the four quadrennials. Among the states, Amapá (12.2) and Sergipe (11.0) had highest rates in 2000-2003, while Paraíba (3.0) and Rio Grande do Sul (3.2) were the lowest. There was declining tendency of hospitalization rates in Brazil (APC= $-4.21 /$ $95 \% \mathrm{Cl}-7.23 ;-1.78$ ), as well as in the Northeast regions (APC $=-4.21 / 95 \% \mathrm{Cl}-7.23 ;-1.78$ ) and Southeast $(\mathrm{APC}=-4.21 /$ $95 \% \mathrm{Cl}-7.23 ;-1.78)$. The others showed a stable trend. Conclusion: Despite the decrease in the number of hospitalizations and declining trend of rates between 2000-2015 in the country, the number of women who need to be hospitalized to end abortion remains high.

Keywords: Abortion, Hospitalization, Trends.

\section{RESUMEN}

Objetivo: Analizar magnitud y tendencias de hospitalizaciones por complicaciones del aborto en Brasil, $2000-2015$. Métodos: Estudio ecológico, de tendencia temporal, con datos de 2000-2015 sobre hospitalizaciones por complicaciones del aborto en mujeres de 15 a 44 años en Brasil, regiones y estados, obtenidos de DATASUS e IBGE. El método Joinpoint se utilizó para evaluar tendencia en las tasas de hospitalización, con determinación de variación porcentual anual (VPA) y intervalos de confianza del 95\% (IC95\%). Resultados: Entre 2000-2015, hubo 3.569.661 hospitalizaciones por aborto en Brasil. La región con tasa más alta fue Nordeste $(7,0)$ en 2000-2003, mientras queSur tuvo tasas más bajas en los cuatro cuadrienios. Entre los estados, Amapá $(12,2)$ y Sergipe $(11,0)$ tuvieron tasas más altas en 2000-2003, mientras que Paraíba $(3,0)$ y Rio Grande do Sul $(3,2)$ más bajas. Hubo tendencia a reducir tasa de hospitalización en Brasil (VPA=4.21/ IC95\% -7.23;-1.78), así como en las regiones del Nordeste (VPA=-4.21/ IC95\% -7.23;-1.78) y Sudeste (VPA=-4.21/ IC 95\% -7.23;-1.78). Los otros mostraron tendencia estable. Conclusión: A pesar de la disminución en hospitalizaciones y tendencia a que las tasas disminuyan entre 2000-2015 en el país, el número de mujeres que necesitan hospitalización para poner fin al aborto sigue siendo alto.

Palabras clave: Aborto, Hospitalización, Tendencias.

1Universidade Estadual do Piauí (UESPI), Teresina - PI. *E-mail: madeiro@uol.com.br SUBMETIDO EM: 7/2020 


\section{INTRODUÇÃO}

De acordo com a Organização Mundial da Saúde (OMS) o aborto inseguro é definido como o procedimento de interrupção de uma gestação realizado por pessoas não habilitadas, em um ambiente sem as condições adequadas (CECATTI JG, 2010). Trata-se de relevante causa de morbidade e mortalidade materna, principalmente em países onde o aborto seguro é indisponível, restrito ou inacessível (TAVARA L, 2014; RASHID S, et al., 2017).

Estima-se que, em todo o mundo, cerca de 20 milhões de mulheres interrompam a gravidez de maneira insegura por ano e que 8,5 milhões delas sofram por complicações resultantes desses procedimentos (SINGH S e MADDOW-ZIMET I, 2016).

No Brasil, um inquérito domiciliar com amostra aleatória de 2.002 mulheres de 18-39 anos estimou que ocorreram meio milhão de abortos em 2015 e que aos 40 anos uma em cada cinco mulheres realizou ao menos um aborto (DINIZ D, et al., 2017).

Desde o início dos anos 1990 houve declínio na morbidade e mortalidade relacionadas ao aborto, provavelmente em decorrência da substituição de métodos inseguros e invasivos pelo uso do misoprostol (ALLEN R e O'BRIEN BM, 2009). Um estudo que analisou a tendência de complicações de aborto no Brasil, entre 1992 a 2009, através dos dados de internações hospitalares obtidos no Sistema de Informações Hospitalares do Sistema Único de Saúde (SIH/SUS), mostrou que o número de mulheres brasileiras tratadas por complicações de aborto caiu de 345.000 em 1992 para 223.000 em 2009, representando declínio de $35,4 \%$ no total e de $2,1 \%$ ao ano.

Durante esse período, o número de mulheres tratadas por complicações menos graves relacionadas ao aborto diminuiu $35 \%$, enquanto que as tratadas por complicações mais graves houve redução de $60 \%$ (SINGH S, et al., 2012).

O número de mulheres que precisam ser internadas para finalizar o aborto ainda permanece alto, sendo a curetagem pós-aborto o segundo procedimento obstétrico mais frequente no SUS (MARTINS-MELO FR, et al., 2012). O misoprostol, considerado mais simples e acessível em relação aos outros meios abortivos, é um medicamento que apresenta eficácia clínica de aproximadamente $85 \%$, quando usado corretamente (ALLEN R e O'BRIEN BM, 2009; MATERNOWSKA MC, et al., 2014).

Entretanto, o conhecimento das mulheres sobre a dosagem e via de administração é frequentemente deficiente e, em consequência, a droga é usada de várias maneiras e nem sempre de forma adequada (ZAMBERLIN N, et al., 2012). Além disso, o medicamento é vendido clandestinamente, sendo sua qualidade e autenticidade questionáveis (DINIZ D e MADEIRO A, 2012).

A ilegalidade do aborto dificulta o conhecimento de sua real magnitude e repercussão, geralmente por conta do estigma relacionado ao tema. Dessa forma, as estimativas indiretas têm sido ferramentas importantes para a obtenção de dados confiáveis, representando alternativa viável do ponto de vista metodológico (SINGH S e MADDOW-ZIMET I, 2016).

Um estudo que analisou os dados de internações hospitalares no Brasil entre 1992 e 2005 registradas no SIH/SUS, aplicando a mesma metodologia do Instituto Alan Guttmacher, com correção dos sub-registros e da proporção referente aos abortos espontâneos. Tal estudo observou redução da estimativa do número de abortos induzidos (de 1.455.283 em 1992 para $1.056 .573 \mathrm{em}$ 2005), da razão de abortos induzidos por 100 nascimentos vivos (de $43 \%$ para $29 \%$ ) e das taxas anuais de abortos induzidos por 100 mulheres de 15 a 49 anos (de 3,69 para 2,07), com diferença regional significativa entre Nordeste e Centro-Oeste, quando comparadas com a região Sul que apresentou menor risco de abortos induzidos (MONTEIRO MFG e ADESSE L, 2006).

Considerando que os dados sobre a prática de aborto induzido no Brasil ainda são escassos, este trabalho pretende atualizar as informações sobre as internações hospitalares. Dessa forma, o objetivo central foi analisar a magnitude e tendência das internações hospitalares por complicações aborto em todo o país, no período de 2000 a 2015. 


\section{MÉTODOS}

Realizou-se estudo observacional descritivo, do tipo ecológico, de série temporal, para analisar as internações hospitalares por complicações de aborto de mulheres de 15 a 44 anos no Brasil, suas regiões e estados, no período de 2000 a 2015.

O estudo do período de 16 anos teve o objetivo de controlar possíveis inconsistências no sistema de notificação dos municípios e estados ao Ministério da Saúde. Foram utilizados dados secundários, disponibilizados em domínio público, tendo como fonte o número de internações hospitalares de mulheres por complicações do aborto, obtidos no SIH/SUS.

A procura na base de dados foi feita através do Código Internacional de Doenças (CID-10), capítulo XV (que trata da Gravidez, Parto e Puerpério), sendo considerados os códigos O03, O04, O05. O número de mulheres residentes foi obtido do Instituto Brasileiro de Geografia e Estatística. Os dados de internações por consequência de aborto foram tabulados, por ano, para cada estado, região e para o país como um todo. Calculou-se a taxa de internações hospitalares por 1.000 mulheres de 15 a 44 anos.

Para cada região foi determinada a tendência das taxas internações por aborto entre 2000 e 2015, utilizando-se o método Joinpoint. Para descrever a tendência linear por período, foram calculadas a variação percentual anual (VPA) e seus respectivos intervalos de confiança de 95\% (IC95\%), compondo uma linha de regressão de acordo com o logaritmo natural das taxas.

A tendência de aumento do número de internações foi considerada quando o valor mínimo do IC95\% se mostrou superior a zero $(>0)$. Por outro lado, caracterizou-se redução quando houve declínio na tendência e o IC95\% foi inferior a zero $(<0)$. A estabilidade da tendência foi definida quando, independentemente da tendência, o IC95\% incluiu o valor zero (KIM HJ, et al., 2000).

O estudo teve como fonte o uso de informações secundárias disponíveis em uma base de dados públicos, não havendo risco de identificação das mulheres ou qualquer relacionado ao uso e divulgação das informações coletadas. Dessa forma, o estudo não necessitou ser submetido à aprovação por Comitê de Ética em Pesquisa para avaliação de aspectos éticos, seguindo recomendação da Resolução CONEP 510/2016 (BRASIL, 2016).

\section{RESULTADOS}

Entre 2000 e 2015 ocorreram 3.569.661 internações por complicações de aborto em mulheres de 15 a 44 anos no Brasil. Durante o período, houve redução do número de internações de 241.694 no ano 2000, para 201.119 em 2015, sendo este o ano que apresentou o menor valor observado (Figura 1).

Figura 1 - Internações hospitalares por complicações de aborto em mulheres de 15 a 44 anos. Brasil, 2000-2015.

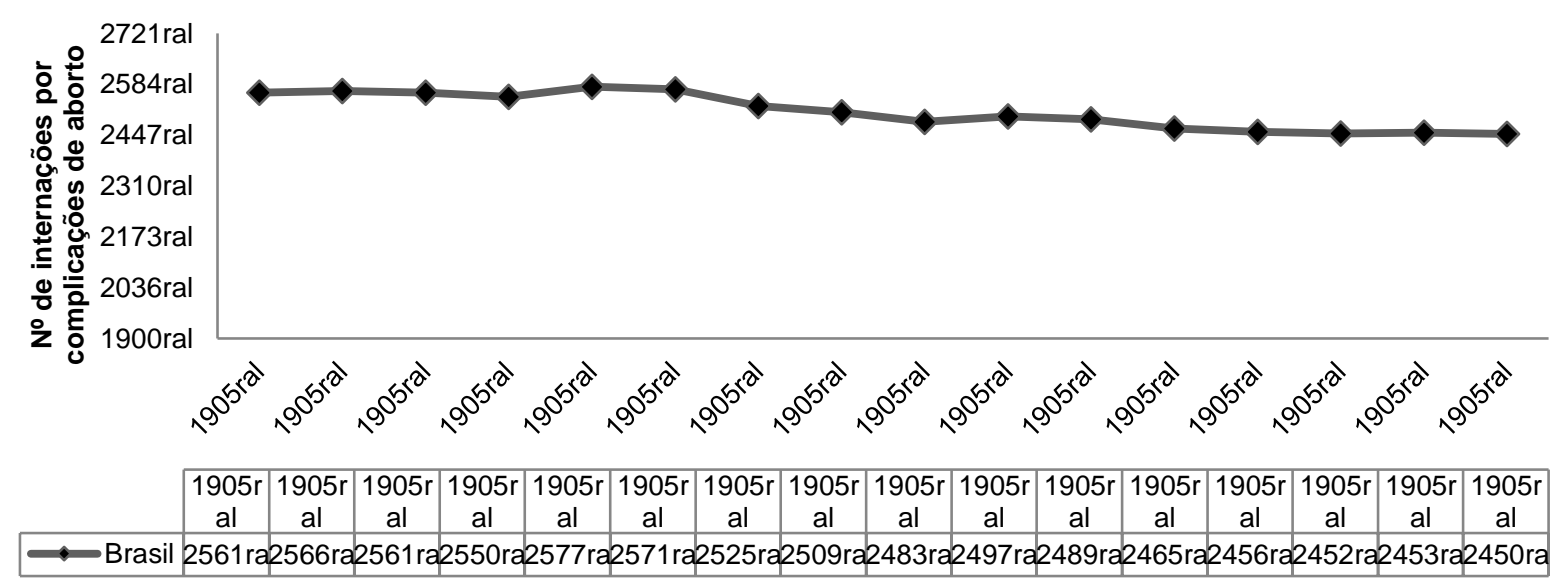

Fonte: Freitas SNB, et al., 2020. Baseado em dados do SIH/SUS. 
Houve declínio das taxas de internações por quadriênio do país como um todo, bem como em todas as regiões. Os quadriênios com as maiores e menores taxas foram, respectivamente, 2000-2003 e 2012-2015. Ocorreu tendência de redução da taxa de internações no país como um todo no período estudado (VPA= $4,21 /$ IC95\% -7,23;-1,78). A região que apresentou a maior taxa de internações por complicações de aborto foi a Nordeste (7,0 internações/ 1.000 mulheres) em 2000-2003, enquanto que a Sul apresentou os menores valores nos quatro quadriênios. As regiões Nordeste (VPA=-3,48/IC95\% -6,34;-1,12) e Sudeste (VPA= -2,82/ IC95\% -3,88;-1,19) apresentaram tendência de redução, tendo as demais evidenciado tendências estáveis (Tabela 1).

Tabela 1 - Taxas de internações hospitalares por complicações de aborto (por 1.000 mulheres) no Brasil e regiões, por quadriênio. Brasil, 2000-2015.

\begin{tabular}{cccccccc}
\hline \multirow{2}{*}{ Regiões } & \multicolumn{3}{c}{ Taxas de internações } & & \multicolumn{2}{c}{ Tendência } \\
\cline { 2 - 4 } \cline { 7 - 8 } & $\mathbf{2 0 0 0 - 2 0 0 3}$ & $\mathbf{2 0 0 4 - 2 0 0 7}$ & $\mathbf{2 0 0 8 - 2 0 1 1}$ & $\mathbf{2 0 1 2 - 2 0 1 5}$ & & VPA & IC95\% $^{2}$ \\
\hline Todas & 5,5 & 5,1 & 4,5 & 4,1 & & $-4,21$ & $-7,23 ;-1,78$ \\
Centro-Oeste & 5,0 & 4,8 & 4,0 & 3,6 & & $-1,87$ & $-3,10 ; 1,83$ \\
Norte & 5,9 & 6,8 & 6,0 & 5,1 & & $-2,27$ & $-3,28 ; 1,03$ \\
Nordeste & 7,0 & 6,4 & 5,4 & 4,8 & & $-3,48$ & $-6,34 ;-1,12$ \\
Sudeste & 5,2 & 4,7 & 4,0 & 3,8 & & $-2,82$ & $-3,88 ;-1,19$ \\
Sul & 3,6 & 3,3 & 3,5 & 3,5 & & $-1,04$ & $-2,77 ; 2,46$ \\
\hline
\end{tabular}

Legenda: ${ }^{1}$ VPA: variação percentual annual; ${ }^{2}$ IC95\%: intervalo de confiança de $95 \%$.

Fonte: Freitas SNB, et al., 2020. Baseado em dados do SIH/SUS e IBGE.

Dentre os estados, Amapá $(12,2)$ e Sergipe $(11,0)$ apresentaram as maiores taxas de internações no quadriênio 2000-2003, enquanto Mato Grosso $(3,3)$ e Rio Grande do Sul $(3,2)$ apresentaram os menores valores. O estado do Amapá se manteve, durante todo período estudado, com as maiores taxas de internações observadas. A maior parte dos estados apresentou tendência de estabilidade nas taxas de internações, porém em 03 deles (Paraíba, Roraima, Tocantins) houve tendência de aumento e em 09 (Alagoas, Bahia, Ceará, Distrito Federal, Minas Gerais, Pernambuco, Piauí, Rio de Janeiro e Sergipe) tendência de queda (Tabela 2).

Tabela 2 - Taxas de internações hospitalares por complicações de aborto (por 1.000 mulheres), por estado e por quadriênio. Brasil, 2000-2015.

\begin{tabular}{|c|c|c|c|c|c|c|}
\hline \multirow{2}{*}{ Estados } & \multicolumn{4}{|c|}{ Taxas de internações } & \multicolumn{2}{|c|}{ Tendência } \\
\hline & 2000-2003 & 2004-2007 & 2008-2011 & $2012-2015$ & VPA $^{1}$ & IC95\% ${ }^{2}$ \\
\hline Acre & 9,2 & 10,2 & 8,4 & 7,4 & $-1,23$ & $-2,46 ; 2,93$ \\
\hline Alagoas & 7,6 & 7,3 & 5,1 & 4,3 & $-2,03$ & $-4,21 ;-1,05$ \\
\hline Amapá & 12,2 & 12,9 & 11,7 & 9,3 & $+1,44$ & $-1,34 ; 2,51$ \\
\hline Amazonas & 6,3 & 8,5 & 6,5 & 4,2 & $-1,70$ & $-3,29 ; 1,88$ \\
\hline Bahia & 9,0 & 7,7 & 6,1 & 5,3 & $-2,13$ & $-3,12 ;-1,08$ \\
\hline Ceará & 7,5 & 6,6 & 5,3 & 4,7 & $-2,04$ & $-2,88 ;-1,12$ \\
\hline Distrito Federal & 8,2 & 8,9 & 5,9 & 4,0 & $-2,31$ & $-3,18 ;-1,29$ \\
\hline Espírito Santo & 5,5 & 4,5 & 3,8 & 3,5 & $-1,88$ & $-2,47 ; 2,02$ \\
\hline Goiás & 4,1 & 4,0 & 3,3 & 3,2 & $+1,04$ & $-2,03 ; 2,87$ \\
\hline Maranhão & 4,3 & 4,9 & 4,6 & 4,8 & $+1,98$ & $-1,38 ; 3,31$ \\
\hline Mato Grosso & 3,3 & 3,6 & 3,7 & 3,9 & $+1,67$ & $-2,48 ; 2,93$ \\
\hline Mato Grosso Sul & 5,4 & 4,6 & 4,0 & 3,9 & $-1,03$ & $-2,90 ; 2,18$ \\
\hline Minas Gerais & 5,2 & 4,4 & 4,2 & 3,9 & $-2,19$ & $-3,81 ;-1,10$ \\
\hline Pará & 5,7 & 5,9 & 5,1 & 4,7 & $-1,04$ & $-3,38 ; 2,08$ \\
\hline Paraíba & 3,0 & 4,6 & 4,9 & 4,4 & $+2,19$ & 1,$21 ; 3,58$ \\
\hline Paraná & 3,6 & 3,3 & 3,2 & 3,5 & $+1,07$ & $-2,14 ; 3,04$ \\
\hline Pernambuco & 6,8 & 6,1 & 5,0 & 4,6 & $-2,43$ & $-4,31 ;-1,11$ \\
\hline Piauí & 7,2 & 6,3 & 5,4 & 4,7 & $-3,12$ & $-4,87 ;-1,24$ \\
\hline Rio de Janeiro & 6,3 & 5,6 & 4,2 & 4,3 & $-2,14$ & $-3,81 ;-1,10$ \\
\hline R. Grande Norte & 4,1 & 3,0 & 4,1 & 4,5 & $+1,32$ & $-2,13 ; 2,89$ \\
\hline R. Grande Sul & 3,2 & 3,1 & 3,5 & 3,3 & $+1,09$ & $-1,88 ; 3,04$ \\
\hline Rondônia & 4,5 & 4,6 & 4,5 & 4,5 & $-1,27$ & $-4,32 ; 2,01$ \\
\hline Roraima & 3,7 & 7,5 & 10,6 & 10,0 & $+2,20$ & 1,$21 ; 4,12$ \\
\hline Santa Catarina & 4,3 & 3,7 & 3,8 & 3,9 & $-1,22$ & $-2,82 ; 2,47$ \\
\hline São Paulo & 4,8 & 4,5 & 3,9 & 3,5 & $-1,45$ & $-1,88 ; 2,35$ \\
\hline Sergipe & 11,0 & 9,2 & 6,9 & 5,5 & $-2,56$ & $-4,31 ;-1,11$ \\
\hline Tocantins & 3,7 & 5,8 & 5,9 & 5,7 & $+2,31$ & 1,$13 ; 3,49$ \\
\hline
\end{tabular}

Legenda: ${ }^{1}$ VPA: variação percentual annual; ${ }^{2}$ IC95\%: intervalo de confiança de $95 \%$.

Fonte: Freitas SNB, et al., 2020. Baseado em dados do SIH/SUS e IBGE. 


\section{DISCUSSÃO}

Os resultados evidenciaram diminuição no número e nas taxas de internações hospitalares tanto para o país como para cada região isoladamente. Embora a maioria dos estados e regiões se mantenha com tendências estáveis, o Brasil apresentou tendência de queda nas taxas de internações.

As regiões Norte e Nordeste obtiveram as maiores taxas de internações, enquanto a Sul, as menores. Apenas três estados (Paraíba, Roraima e Tocantins) apresentaram tendência de aumento das taxas. Esses dados corroboram o padrão de redução no número de internações para tratamento de complicações do aborto observado desde a década de 1990 no país (SINGH S, et al., 2012; MONTEIRO MFG, et al., 2015).

Em um estudo que avaliou o número de interações por complicações de aborto no Brasil por região e por faixa etária entre 1995 e 2013 constatou diminuição nas taxas de aborto induzido por 1.000 mulheres de 15 a 49 anos em todas as regiões do país. As regiões que apresentaram maior número de internações foram Sudeste e Nordeste. Ambas obtiveram redução importante no número de internações durante o período: 27 e 35\%, respectivamente. Com relação às taxas de aborto induzido por faixa etária, observou-se maior redução na faixa de 40 a 49 anos (50\%), seguida de 20 a 29 anos (49\%) (MONTEIRO MFG, et al., 2015).

Outras investigações nesse período também evidenciaram queda nas internações por aborto no país. Entre 2008 e 2015 houve diminuição de 9,5\% no número de internações por procedimentos relacionados ao aborto, com média de 200.000 internações por ano (CARDOSO BB, et al., 2020). Já no presente estudo, os valores variaram de 247.621 (2004) a 201.119 (2015).

A Pesquisa Nacional de Aborto (PNA) de 2016, por sua vez, mediante inquérito domiciliar com amostra aleatória de mulheres entre 18 e 39 anos estimou que, somente no ano de 2015, aproximadamente 503.000 mulheres realizaram aborto e cerca de metade (48\%) precisou de internação hospitalar (DINIZ D, et al., 2017).

A análise das taxas de internações entre estados, no atual estudo, evidenciou algumas diferenças importantes. O Nordeste, por exemplo, abrange estados que apresentaram reduções nas taxas de internações por 1.000 mulheres, como Sergipe com valores variando de 11,0 em 2000-2003 a 5,5 em 20122015, mas também engloba o estado da Paraíba que apresentou tendência de aumento das taxas durante o período.

A análise do número de abortos inseguros por estimativas indiretas de dados do SIH/SUS entre 19922012 também evidenciou heterogeneidade das estimativas entre unidades federativas e sugere que as diferenças regionais podem ser atribuídas ao maior acesso e adesão aos métodos contraceptivos nos estados com menores taxas de aborto inseguro (MARTINS-MELO FR, et al., 2012).

Duas pesquisas conduzidas em estados do Nordeste, que utilizaram dados de internações hospitalares para estimar a magnitude do aborto inseguro, mostraram resultados semelhantes a este estudo. Em Pernambuco, entre 1996-2006, observou-se número elevado de internações por aborto, com estimativa de 621.022 abortos inseguros e redução de $7,7 \%$ dos casos entre os extremos da série histórica (MELLO FMB, et al., 2011).

Já no Piauí, entre 2000-2010, ocorreu identificação de 55.678 internações por complicações de aborto no período analisado, porém com decréscimo de 35\% (10.362 em 2000 para 6.738 em 2010) e tendência de queda de 2,2\% ao ano (MADEIRO A, et al., 2015). Vários fatores podem ter contribuído para o declínio das internações hospitalares para tratamento de complicações relacionadas ao aborto. Até os anos 1980, sondas, substâncias cáusticas ou objetos perfurantes eram métodos abortivos bastante utilizados (CORRÊA MCDV e MASTRELLA M, 2012). O misoprostol (Cytotec), droga introduzida para tratamento de úlcera gástrica, teve seu uso aprovado em 1985 e comercialização a partir de 1988.

O medicamento foi logo percebido em razão de seu potencial abortivo e, em 1991, sua venda livre proibida, passando a exigir receita médica com retenção da prescrição. Já em 1998, foi incluído na lista de fármacos sujeitos a controle especial, conferindo restrição à droga e seu uso abortivo (ARILHA MM, 2012).

Desde a década de 1990, o misoprostol tem se apresentado como o método abortivo preferencial entre as mulheres brasileiras, talvez por ser mais economicamente viável e menos inseguro (BARBOSA RM e ARILHA 
M, 1993). Há evidências de que a droga é, hoje, o principal meio para se induzir aborto em mulheres no país (SINGH S e WULF D, 1994). Sua chegada coincide com menor número de casos mais graves de complicações, principalmente infecciosas (FAÚNDES A, et al., 1996).

Um estudo utilizando dados retrospectivos em uma maternidade entre 1988-1992 demonstrou que as infecções eram muito menos comuns entre pacientes pós-aborto que usaram misoprostol do que entre aquelas com uso de outros métodos (ARILHA MM, 2012).

Apesar da restrição oficial ao medicamento, as mulheres conseguem obtê-lo comprando-o no mercado clandestino, porém submetendo-se ao risco de uso de medicamentos falsificados, com pouca ou nenhuma informação sobre as indicações sobre dose e formas de administração da droga (DINIZ D e MADEIRO, 2012). A Organização Mundial de Saúde recomenda o misoprostol para o abortamento medicamentoso (800mcg vaginal ou sublingual a cada 8 horas até três doses) (ORGANIZAÇÃO MUNDIAL DE SAÚDE, 2012).

As mulheres utilizam a droga de vários modos, com doses variando entre 4 e 16 comprimidos, e até doses mais altas em alguns casos. Também são variáveis os intervalos entre doses e a via de administração, geralmente, sendo utilizada a via vaginal ou a oral e até mesmo uma combinação de ambas (ZAMBERLIN N, et al., 2012).

Além da redução no uso de métodos abortivos mais invasivos, os serviços de aborto legal, mesmo que de forma insuficiente, também podem ter contribuído para a redução das internações por complicações de aborto. Um estudo de abrangência nacional nos serviços de aborto previsto em leientre 2013-2015, avaliou 68 serviços, em uma etapa censitária, com questionário eletrônico, e uma etapa amostral e presencial, em 5 serviços de referência.

De acordo com a pesquisa, o principal motivo para o aborto legal foi o estupro (94\%) e as principais dificuldades encontradas no atendimento das mulheres relacionaram-se ao desconhecimento dos profissionais de saúde sobre a legislação vigente, o receio de serem punidos legalmente, a influência dos valores ético-religiosos e o temor do estigma de serem conhecidos como aborteiros. Apesar dessas barreiras, houve melhora na qualidade do atendimento inicial à mulher, tanto na oferta de contracepção de emergência e profilaxia de doenças sexualmente transmissíveis como na menor exigência de documentos desnecessários para a realização do aborto (MADEIRO AP e DINIZ D, 2016).

Também é preciso considerar o aumento do uso de métodos anticoncepcionais, ao longo das últimas décadas, como fator decisivo para o declínio da fecundidade no país e, consequentemente, para a queda nas taxas de internações por aborto. Tanto o acesso como o conhecimento sobre métodos mais eficazes e seguros aumentaram no país (BRANDT GP, et al., 2018).

Os dados da Pesquisa Nacional sobre Demografia e Saúde (PNDS) de 1996 e 2006, para mulheres entre 15 a 44 anos, mostraram aumento no uso de métodos contraceptivos hormonais e da esterilização masculina em todas as classes econômicas, além da diminuição da esterilização feminina, método mais utilizado por mulheres de baixa escolaridade (BRASIL, 2009). Por sua vez, as dificuldades de informação e acesso aos métodos anticoncepcionais estão relacionadas às condições socioeconômicas e, muitas vezes, o aborto induzido pode ser utilizado como um método de planejamento familiar para as mulheres em situação de vulnerabilidade (CECATTI JG, et al., 2010). Além disso, as diferenças nas taxas de complicações de aborto entre localidades, nos estudos com dados do SIH/SUS, também podem estar associadas à maior oferta e acesso aos serviços do SUS.

Uma pesquisa que analisou internações por abortos no estado de São Paulo em 1995 observou que a concentração de mulheres usuárias do SUS na cidade de São Paulo era maior que em municípios do interior e apontou este como um dentre outros fatores para as diferentes proporções de aborto em cidades do mesmo estado (SORRENTINO SR e LEBRÃO ML, 1998).

Um estudo baseado nos dados da Pesquisa Nacional sobre Acesso, Utilização e Promoção do Uso Racional de Medicamentos (PNAUM), analisou a prevalência do uso atual de contraceptivos orais e injetáveis por mulheres brasileiras de 15 a 49 anos, segundo variáveis demográficas, socioeconômicas e aspectos 
relacionados ao acesso a esses medicamentos entre setembro e fevereiro (2013-2014), em 20.404 domicílios urbanos brasileiros.

Os dados evidenciaram predomínio dos contraceptivos orais em relação aos mensais ou trimestrais e maior prevalência de ambos nas faixas etárias entre 20 e 29 anos. Além disso, o consumo de contraceptivos orais foi maior na região Sul e menor no Norte, mas não houve diferença significativa entre as regiões com relação aos dos injetáveis (FARIAS MR, et al., 2016).

A PNDS (1996 e 2006) também evidenciou queda na proporção de indesejabilidade do último filho nascido nos cinco anos anteriores à pesquisa (de 23,1\% para 18,2\%) e de gravidez em curso (de 28,2\% para 19,0\%), havendo maior prevalência de nascimentos não desejados entre as mulheres mais pobres, menos escolarizadas. Quanto ao comportamento etário, a distribuição da fecundidade mostrou redução da taxa específica do grupo de 15 a 19 anos, com 93 nascimentos por mil mulheres nessa faixa etária, em 2000 e 71 , em 2010 (BERQUÓ ES e CAVENAGHI SM, 2014).

Este estudo tem pelo menos duas limitações que devem ser consideradas. Primeiro, as internações por complicações de aborto declaradas pelo DATASUS representam somente as mulheres que tiveram complicações e foram atendidas na rede pública, subestimando a magnitude da prática. Não se sabe sobre as mulheres atendidas na rede privada e tampouco sobre aquelas que não necessitaram de hospitalização após o aborto.

Segundo a utilização de dados secundários pode apresentar inconsistências na qualidade das informações, principalmente em relação à causa da internação nos diferentes estados do país. Levando em consideração que a base de dados utilizada neste estudo foi originalmente criada com a finalidade de pagamento, ainda há insuficiência de informações diagnósticas e clínicas das internações, notadamente nas regiões Norte e Nordeste (MACHADO JP, et al., 2016).

A legislação restritiva ao aborto no país dificulta a obtenção de dados reais sobre sua magnitude e repercussão. Além disso, o aborto envolve aspectos de cunho moral e religioso, sendo objeto de forte sanção social. Assim, uma das grandes dificuldades encontradas na realização de pesquisas sobre aborto é a possibilidade de omissão da sua realização por parte das mulheres, tanto pelo estigma associado à prática, como pelo receio da denúncia.

Diante das dificuldades para obtenção de dados, as estimativas indiretas têm se mostrado importantes, apresentando a vantagem de serem viáveis do ponto de vista financeiro e metodológico, como os estudos que utilizam informações de internações hospitalares para procedimentos médicos relacionados à prática do aborto.

\section{CONCLUSÃO}

Apesar de as internações por aborto terem diminuído significativamente no país durante o período analisado, ainda é elevado o número de mulheres com necessidade de hospitalização por complicações do aborto. Esse fato evidencia que muitas mulheres brasileiras não têm suas demandas reprodutivas atendidas, com frequência alta de gestações indesejadas e decisão pela interrupção da gravidez, geralmente de maneira insegura. A oferta de saúde reprodutiva integral, incluindo a anticoncepção auto-decidida e a maternidade voluntária, deve ser estratégia a ser atingida para proteção de todas as mulheres.

\section{REFERÊNCIAS}

1. ALLEN R, O'BRIEN BM. Uses of misoprostol in obstetrics and gynecology. Reviews in Obstetrics \& Gynecology, 2009;2(3):159-168.

2. ARILHA MM. Misoprostol: percursos, mediações e redes sociais para o acesso ao aborto medicamentoso em contextos de ilegalidade no Estado de São Paulo. Ciência \& Saúde Coletiva, 2012; 17(7): 1785-1794.

3. BARBOSA RM, ARILHA M. The Brazilian experience with Cytotec. Studies in Family Planning, 1993; 24(4): $236-240$.

4. BERQUÓ ES, CAVENAGHI SM. Notas sobre os diferenciais educacionais e econômicos da fecundidade no Brasil. Revista Brasileira de Estudos Populacionais, 2014; 31(2): 471-482.

5. BRANDT GP, et al. Anticoncepcionais hormonais na atualidade: um novo paradigma para o planejamento familiar. Revista Gestão \& Saúde, 2018; 18(1):54-62. 
6. BRASIL. Ministério da Saúde. Pesquisa Nacional de Demografia e Saúde da Criança e da Mulher - PNDS 2006: dimensões do processo reprodutivo e da saúde da criança. Brasília: Ministério da Saúde; 2009.

7. BRASIL. Resolução nํ510, de 7 de abril de 2016. Conselho Nacional de Saúde, do Ministério da Saúde. Diário Oficial da União 2016.

8. CARDOSO BB, et al. Aborto no Brasil: o que dizem os dados oficiais? Cadernos de Saúde Pública, 2020; 36(suppl 1):e00188718.

9. CECATTI JG, et al. Aborto no Brasil: um enfoque demográfico. Revista Brasileira de Ginecologia e Obstetrícia, 2010;32(3):105-111.

10. CORREA MCDV, MASTRELLA M. Aborto e misoprostol: usos médicos, práticas de saúde e controvérsia científica. Ciência \& Saúde Coletiva, 2012; 17(7): 1777-1784.

11. DINIZ D, MADEIRO A. Cytotec e aborto: a polícia, os vendedores e as mulheres. Ciência \& Saúde Coletiva, 2012;17(7):1795-1804.

12. DINIZ D, et al. Pesquisa Nacional de Aborto 2016. Ciência \& Saúde Coletiva, 2017; 22(2): 653-660.

13. FARIAS MR, et al. Use of and access to oral and injectable contraceptives in Brazil. Revista de Saúde Pública, 2016; 50(supl 2):14s.

14. FAÚNDES $A$, et al. Postabortion complications after interruption of pregnancy with misoprostol. Advances in Contraception, 1996; 12(1): 1-9.

15. KIM HJ, et al. Permutation tests for joinpoint regression with applications to cancer rates. Statistics in Medicine, 2000; 19(3): 335-351.

16. MACHADO JP, et al. Qualidade da base de dados hospitalares no Brasil: alguns elementos. Revista Brasileira de Epidemiologia, 2016; 19(3): 567-581.

17. MADEIRO A, et al. Estimativas e tendências de aborto provocado no Piauí: um estudo ecológico no período de 2000 2010. Revista Brasileira em Promoção da Saúde, 2015; 28(2):168-175.

18. MADEIRO AP, DINIZ D. Serviços de aborto legal no Brasil - um estudo nacional. Ciência \& Saúde Coletiva, 2016; 21(2): 563-572.

19. MARTINS-MELO FR, et al. Temporal trends and spatial distribution of unsafe abortion in Brazil, 1996-2012. Revista de Saúde Pública, 2014;48(3):508-520.

20. MATERNOWSKA MC, et al. Perceptions of misoprostol among providers and women seeking post-abortion care in Zimbabwe. Reproductive Health Matters, 2014;22(sup44):16-25.

21. MELLO FMB, et al. Magnitude do aborto inseguro em Pernambuco, Brasil, 1996-2006. Cadernos de Saúde Pública, $2011 ; 27(1): 87-93$.

22. MONTEIRO MFG, ADESSE L. Estimativas de aborto induzido no Brasil e Grandes Regiões (1992-2005). Revista de Saúde Sexual e Reprodutiva, 2006; 26: 1-10.

23. MONTEIRO MFG, et al. Atualização das estimativas da magnitude do aborto induzido, taxas por mil mulheres e razões por 100 nascimentos vivos do aborto induzido por faixa etária e grandes regiões. Brasil, 1995 a 2013. Reprodução \& Climatério, 2015; 30: 11-18.

24. ORGANIZAÇÃO MUNDIAL DE SAÚDE. Aborto Seguro: Orientação Técnica e Política para os Sistemas de Saúde. $2^{\underline{a}}$ edição. Genebra: OMS; 2012.

25. RASHID S, et al. Evaluating implementation of the World Health Organization's Strategic Approach to strengthening sexual and reproductive health policies and programs to address unintended pregnancy and unsafe abortion. Reproductive Health, 2017; 14(1): 153.

26. SINGH S, WULF D. Estimated levels of induced abortion in six Latin American countries. International Family Planning Perspectives, 1994; 20(1): 4-13.

27. SINGH S, et al. Trends in hospitalization for abortion-related complications in Brazil, 1992-2009: why the decline in numbers and severity? International Journal of Gynecology and Obstetrics, 2012; 118(suppl. 2): S99-106.

28. SINGH S, MADDOW-ZIMET I. Facility-based treatment for medical complications resulting from unsafe pregnancy termination in the developing world, 2012: a review of evidence from 26 countries. British Journal of Obstetrics and Gynecology, 2016; 123(9): 1489-1498.

29. SORRENTINO SR, LEBRÃO ML. Os abortos no atendimento hospitalar do estado de São Paulo, 1995. Revista Brasileira de Epidemiologia, 1998; 1(3): 256-267.

30. TAVARA L. Contribution of obstetrics and gynecology societies in South America to the prevention of unsafe abortion in the region. International Journal of Gynecology and Obstetrics, 2014; 126: S7-9.

31. ZAMBERLIN N, et al. Latin American women's experiences with medical abortion in settings where abortion is legally restricted. Reproductive Health, 2012; 9(1): 34. 